\title{
Using ages and kinematic traceback: the origin of young local associations
}

\author{
David Fernández, Francesca Figueras and Jordi Torra \\ Departament d'Astronomia i Meteorologia, IEEC-Universitat de Barcelona \\ Av. Diagonal 647, E-08028 Barcelona, Spain \\ email: david.fernandez@am.ub.es
}

\begin{abstract}
Over the last decade, several groups of young (mainly low-mass) stars have been discovered in the solar neighbourhood (closer than $\sim 100 \mathrm{pc}$ ), thanks to cross-correlation between $\mathrm{X}$-ray, optical spectroscopy and kinematic data. These young local associations - including an important fraction whose members are Hipparcos stars - offer insights into the star formation process in low-density environments, shed light on the substellar domain, and could have played an important role in the recent history of the local interstellar medium. Ages estimates for these associations have been derived in the literature by several ways (HR diagram, spectra, Li and $\mathrm{H} \alpha$ widths, expansion motion, etc.). In this work we have studied the kinematic evolution of young local associations and their relation to other young stellar groups and structures in the local interstellar medium, thus casting new light on recent star formation processes in the solar neighbourhood. We compiled the data published in the literature for young local associations, including the astrometric data from the new Hipparcos reduction. Using a realistic Galactic potential we integrated the orbits for these associations and the Sco-Cen complex back in time. Combining these data with the spatial structure of the Local Bubble and the spiral structure of the Galaxy, we propose a recent history of star formation in the solar neighbourhood. We suggest that both the Sco-Cen complex and young local associations originated as a result of the impact of the inner spiral arm shock wave against a giant molecular cloud. The core of the giant molecular cloud formed the Sco-Cen complex, and some small cloudlets in a halo around the giant molecular cloud formed young local associations several million years later. We also propose a supernova in young local associations a few million years ago as the most likely candidate to have reheated the Local Bubble to its present temperature.
\end{abstract}

Keywords. Galaxy: kinematics and dynamics, Galaxy: solar neighbourhood, Galaxy: open clusters and associations: general, Stars: kinematics, Stars: formation, ISM: individual objects: Local Bubble

\section{Introduction}

In this work we propose a scenario for the history of the recent star formation (during the last 20-30 Myr) in the nearest solar neighbourhood ( $150 \mathrm{pc})$, from the study of the spatial and kinematic properties of the members of the so-called young local associations, the Sco-Cen complex and the Local Bubble, the most important structure observed in the local interstellar medium (ISM).

\section{The local interstellar medium: the Local Bubble}

Locally, within the nearest 100 pc, the ISM is dominated by the Local Bubble (LB). The displacement model (Snowden et al. 1998) for this structure assumes that the irregular local HI cavity is filled by an X-ray-emitting plasma, with an emission temperature of $\sim 10^{6} \mathrm{~K}$ and a density, $n_{e}$, of $\sim 0.005 \mathrm{~cm}^{-3}$. Snowden et al. (1998) derived an extension for the LB of 40 to $130 \mathrm{pc}$, it being larger at higher Galactic latitudes and smaller nearer 
the equator. Lallement et al. (2003) obtained the contours of the LB from NaI absorption measurements, tracing them with an estimated precision of $\approx \pm 20 \mathrm{pc}$ in most directions.

Several models have been presented to explain the origin of the LB. The consensus is reached with a scenario in which about 10-20 supernovae (SNe) formed the local cavity and, after that, a few SNe reheated the LB a few Myr ago, explaining the currently observed temperature of the diffuse soft X-ray background (Breitschwerdt \& Cox 2004). Some authors have remarked that there is independent evidence for the occurrence of a close SN ( $\sim 30 \mathrm{pc}) \sim 5 \mathrm{Myr}$ ago (Knie et al. 1999), which could be the best candidate for reheating the LB. Maíz-Apellániz (2001) proposed that the 2 or 3 SNe that reheated the local cavity could have exploded in LCC (one of the OB associations of the Sco-Cen complex), but his results faced some geometrical problems due to the peripheral situation of LCC with respect to the LB.

\section{Local associations of young stars}

A decade ago, very few PMS stars had been identified less than 100 pc from the Sun. Nearly all the youngest stars $(\leqslant 30 \mathrm{Myr})$ studied then were located more than $140 \mathrm{pc}$ away in the molecular clouds of Taurus, Chamaeleon, Lupus, Sco-Cen and R CrA. The cross-correlation of the Hipparcos and ROSAT catalogues suddenly changed this; a few stars were identified as very young but closer than $100 \mathrm{pc}$, where there are no molecular clouds with stellar forming regions (Neuhäuser \& Brandner 1998). Two explanations for the existence of these young stars far away from SFR were proposed. Sterzik \& Durisen (1995) suggested that the stars were formed in molecular clouds and later ejected as high-velocity stars during the decay of young multiple star systems. Feigelson (1996) suggested that the stars were formed inside small molecular clouds (or cloudlets), which later dispersed among the ISM and therefore can no longer be detected.

These young nearby stars were grouped into clusters, associations and moving groups, each with a few dozen members. Different approaches were used in each YLA discovery, but most of them made use of Hipparcos proper motions, X-ray emission, infrared emission and ground-based spectroscopy and photometry. We have compiled all the published YLA data. Astrometric data come from the new Hipparcos reduction (van Leeuwen 2007). Table 1 shows the mean spatial and kinematic properties, ages and number of members for each YLA. The adopted age shown in Table 1 is that assigned for back-tracing the association orbits in the next section. In Fig. 1 the observational errors for the stars of our compilation are shown.

\section{Ages and integration of orbits}

The integration back in time of the Sco-Cen and YLA orbits allows us to study their origin and possible influence on the local ISM over the last million years. To compute the stellar orbits back in time we used the code developed by Asiain et al. (1999) based on the integration of the equations of motion using a realistic model of the Galactic gravitational potential. We decomposed this potential into three components: the general axisymmetric potential $\Phi_{\mathrm{AS}}$, the potential due to the spiral structure of the Galaxy $\Phi_{\mathrm{Sp}}$, and that due to the central bar $\Phi_{\mathrm{B}}$. Details on the method can be found in Fernández et al. (2008).

\subsection{Orbits of individual stars: are we able to derive dynamic ages?}

One of the most interesting results that could be derived from the study of the past trajectories of the individual members of the young local associations is the direct 
Table 1. Mean spatial coordinates and heliocentric velocity components of the young local associations and the Sco-Cen complex (in the latter case, data from de Zeeuw 1999 (Z99), Madsen et al. 2002 (M02) and Sartori et al. 2003 (S03)). In brackets, the standard deviation of the sampling distribution. $N$ is the number of known members in each association $\left(N_{\mathrm{k}}\right.$ with complete kinematic data).

\begin{tabular}{|c|c|c|c|c|c|c|c|c|c|c|c|}
\hline \multicolumn{2}{|c|}{ Association } & $\begin{array}{r}\overline{\xi^{\prime}} \\
(\mathrm{pc})\end{array}$ & $\begin{array}{r}\overline{\eta^{\prime}} \\
(\mathrm{pc})\end{array}$ & $\begin{array}{r}\overline{\zeta^{\prime}} \\
(\mathrm{pc})\end{array}$ & $\begin{array}{r}\bar{r} \\
(\mathrm{pc})\end{array}$ & $\left(\begin{array}{r}\bar{U} \\
\left(\mathrm{~km} \mathrm{~s}^{-1}\right)\end{array}\right.$ & $\begin{array}{r}\bar{V} \\
\left(\mathrm{~km} \mathrm{~s}^{-1}\right)\end{array}$ & $\begin{array}{r}\bar{W} \\
\left(\mathrm{~km} \mathrm{~s}^{-1}\right)\end{array}$ & $\begin{array}{r}\text { Age } \\
(\mathrm{Myr})\end{array}$ & $N$ & $N_{\mathrm{k}}$ \\
\hline \multicolumn{2}{|c|}{$\begin{array}{l}\text { TW Hya } \\
\text { Tuc-Hor/GAYA } \\
\beta \text { Pic-Cap } \\
\epsilon \text { Cha } \\
\eta \text { Cha } \\
\text { HD } 141569 \\
\text { Ext. R CrA }\end{array}$} & $\begin{array}{l}-18_{(12)} \\
-12_{(26)} \\
-10_{(26)} \\
\left.-52_{(} \quad 3\right) \\
-31_{(3)} \\
\left.-77_{(} \quad 3\right) \\
-81_{(33)}\end{array}$ & $\begin{array}{l}-51_{(16)} \\
-26_{(12)} \\
-7_{(13)} \\
\left.-89_{(\quad} 5\right) \\
-75 \\
\left.10_{(} \quad 8\right) \\
-6(6)\end{array}$ & $\begin{aligned} & \left.22_{(} \quad 7\right) \\
- & 36_{(13)} \\
- & 14_{(10)} \\
- & \left.27_{(} \quad 2\right) \\
- & 32 \\
& \left.64_{(} \quad 8\right) \\
- & \left.28_{(} \quad 9\right)\end{aligned}$ & $\begin{array}{r}60_{(18)} \\
53_{(17)} \\
37_{(13)} \\
107_{(\quad 6)} \\
88 \\
\left.101_{(} \quad 8\right) \\
87_{(33)}\end{array}$ & $\begin{array}{l}-8.4_{(3.9)} \\
-11.4_{(7.3)} \\
-10.1_{(3.5)} \\
-10.2_{(0.8)} \\
-10.1 \\
-5.4_{(1.5)} \\
-2.2_{(6.1)}\end{array}$ & $\begin{array}{l}-18.3_{(3.4)} \\
-22.4_{(5.5)} \\
-15.2_{(3.7)} \\
-18.9_{(0.6)} \\
-18.4 \\
-15.6_{(2.6)} \\
-15.8_{(1.4)}\end{array}$ & $\begin{array}{l}-4.9_{(1.8)} \\
-2.7_{(3.7)} \\
-10.2_{(2.9)} \\
-10.2_{(2.0)} \\
-10.0 \\
-4.4_{(0.8)} \\
-10.9_{(0.8)}\end{array}$ & $\begin{array}{r}8 \\
20 \\
12 \\
10 \\
10 \\
5 \\
13\end{array}$ & $\begin{array}{r}39 \\
52 \\
33 \\
16 \\
18 \\
5 \\
59\end{array}$ & $\begin{array}{r}5 \\
28 \\
21 \\
4 \\
1 \\
2 \\
2\end{array}$ \\
\hline US & $\begin{array}{l}\text { Z99 } \\
\text { M02 } \\
\text { S03 }\end{array}$ & $\begin{array}{l}-141_{(34)} \\
-138_{(27)}\end{array}$ & $\begin{array}{l}-22_{(11)} \\
-22_{(10)}\end{array}$ & $\begin{array}{l}50_{(16)} \\
49_{(12)}\end{array}$ & $\begin{array}{l}145_{(2)} \\
149_{(28)}\end{array}$ & $\begin{array}{l}-0.9 \\
-6.7_{(5.9)}\end{array}$ & $\begin{array}{l}-16.9 \\
-16.0_{(3.5)}\end{array}$ & $\begin{array}{l}-5.2 \\
-8.0(2.7)\end{array}$ & $\begin{array}{r}5-6 \\
8-10\end{array}$ & $\begin{array}{l}120 \\
120 \\
155\end{array}$ & \\
\hline $\mathrm{UCL}$ & $\begin{array}{l}\text { Z99 } \\
\text { M02 } \\
\text { S03 }\end{array}$ & $\begin{array}{l}-122_{(30)} \\
-121_{(26)}\end{array}$ & $\begin{array}{l}-69_{(26)} \\
-68_{(21)}\end{array}$ & $\begin{array}{l}32_{(16)} \\
32_{(15)}\end{array}$ & $\begin{array}{l}140_{(2)} \\
145_{(24)}\end{array}$ & $\begin{array}{l}-7.9 \\
-6.8_{(4.6)}\end{array}$ & $\begin{array}{l}-19.0 \\
-19.3_{(4.7)}\end{array}$ & $\begin{array}{l}-5.7 \\
-5.7_{(2.5)}\end{array}$ & $14-15$ & $\begin{array}{l}221 \\
218 \\
262\end{array}$ & \\
\hline $\mathrm{LCC}$ & $\begin{array}{l}\text { Z99 } \\
\text { M02 } \\
\text { S03 }\end{array}$ & $\begin{array}{l}-62_{(18)} \\
-61_{(14)}\end{array}$ & $\begin{array}{l}-102_{(24)} \\
-100_{(15)}\end{array}$ & $\begin{array}{l}14_{(16)} \\
14_{(15)}\end{array}$ & $\begin{array}{l}118_{(\quad 2)} \\
120_{(18)}\end{array}$ & $\begin{array}{l}-11.8 \\
-8.2(5.1)\end{array}$ & $\begin{array}{l}-15.0 \\
-18.6_{(7.3)}\end{array}$ & $\begin{array}{l}-6.7 \\
-6.4_{(2.6)}\end{array}$ & $11-12$ & $\begin{array}{l}180 \\
179 \\
192\end{array}$ & \\
\hline
\end{tabular}

1 M02 derived an internal velocity dispersion of $1.33 \mathrm{~km} \mathrm{~s}^{-1}$ for US, $1.23 \mathrm{~km} \mathrm{~s}^{-1}$ for UCL and $1.13 \mathrm{~km} \mathrm{~s}-1$ for LCC.

determination of their age. This could be achieved if one observes a clear spatial concentration of the members of one specific association in the past.

However, we have not found this concentration in the past. We have computed the trajectories back in time for the stars of the three associations with the largest number of members with complete, high-quality data. In Fig. 2 we show the spatial dispersion of these stars as a function of time in the past, the results are those shown in the figure. As can be seen, during the last 4 million years, the spatial dispersion remains similar to that observed at present, but it grows rapidly to the past. So, it seems that the combination of the observational errors and the intrinsic velocity dispersion prevent us from deriving the ages for the associations. Or maybe there are some problems with the membership assignment of the stars to each association. Then, in our analysis we will only work with the past trajectories of the associations as a whole.
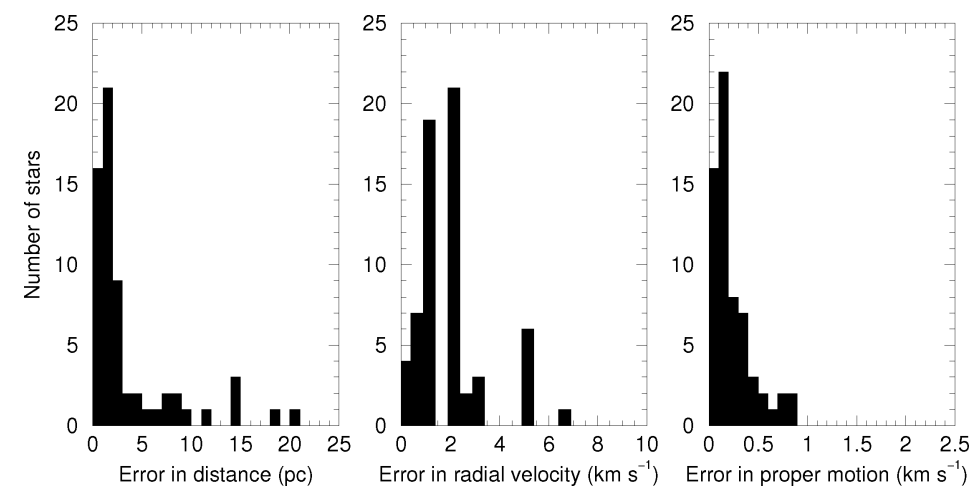

Figure 1. Observational errors in distance (left), radial velocity (center) and tangential velocity (right) for the stars of our compilation of YLA. 


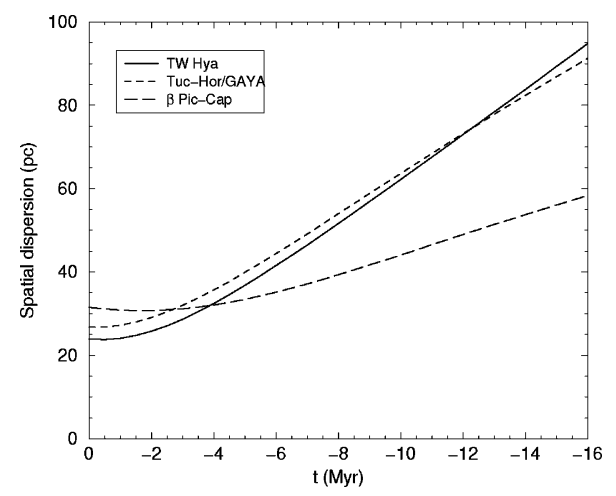

Figure 2. Spatial dispersion of those stars belonging to TW Hya, Tuc-Hor/GAYA and $\beta$ Pic-Cap during the last Myr.

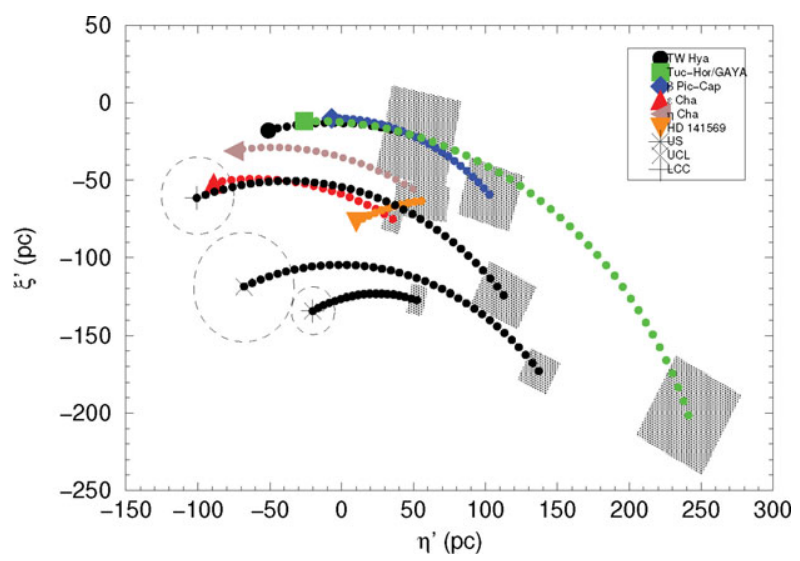

Figure 3. Positions and orbits in the Galactic plane $\left(\xi^{\prime}, \eta^{\prime}\right)$ of YLA and the Sco-Cen complex going back in time to their individual ages. The grey areas show the expected positional errors at birth due to kinematic observational errors. The centre of the $\left(\xi^{\prime}, \eta^{\prime}\right)$ coordinate system is comoving with the LSR.

\subsection{The origin of young local associations}

We have integrated the orbits of the associations using the mean position and velocity for each association (Table 1). The results are presented in Fig. 3. We show the estimated error in the position of the associations at birth (grey area). An error in age shall be read as a displacement of the error areas along the plotted orbits in the figure. The most obvious trend observed is the spatial concentration of all the associations (Sco-Cen complex and YLA) in the first Galactic quadrant in the past. There is a very conspicuous spatial grouping at the time of birth of TW Hya, $\epsilon$ Cha, $\eta$ Cha and HD 141569 (in a sphere $25 \mathrm{pc}$ in radius). At its birth, $\beta$ Pic-Cap was located about $50 \mathrm{pc}$ from the other YLA. The region where these associations were formed had a size of $50 \times 70 \times 40 \mathrm{pc}\left(\xi^{\prime} \mathrm{x}\right.$ $\left.\eta^{\prime} \mathrm{x} \zeta^{\prime}\right)$. At present the volume has increased by a factor of $\sim 3.5$. The errors associated with the mean velocity components of the associations do not have a crucial influence on the previous results: the error areas have typical side lengths of about 10-30 pc.

Mamajek et al. (2000) found that extrapolating past motions (assuming linear ballistic trajectories) shows that TW Hya, $\epsilon$ Cha, $\eta$ Cha and the three subgroups of the ScoCen complex were closest together about 10-15 Myr ago. They suggest that these three 


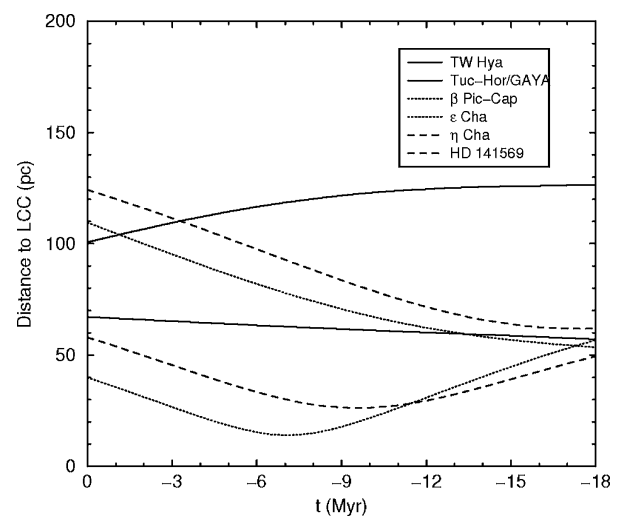

Figure 4. Distances between the centres of YLA and the centre of LCC as a function of time, using the Sco-Cen kinematics in S03.

associations were formed in the progenitor Sco-Cen GMC or in short-lived molecular clouds formed by Sco-Cen superbubbles. Jilinski et al. (2005) locate the birthplaces of $\epsilon$ Cha and $\eta$ Cha at the edge of LCC. Ortega et al. (2004) suggest that the $\beta$ Pic moving group was formed near Sco-Cen, probably due to a SN in this complex. Our compendium of all the known members of the whole set of YLA and the integration of their orbits back in time allow us to present here a more detailed analysis of the origin of YLA.

In Fig. 4 the temporal evolution of the distances between each one of the YLA and the centre of LCC (the nearest OB association) is shown. The instant when the distance minima between LCC and YLA occurred is of great interest. In the cases of the $\eta$ Cha cluster and the $\epsilon$ Cha association, minima with distances of 14 and $26 \mathrm{pc}$, respectively, are obtained for $t \sim-(7-9)$ Myr. No clear distance minima to LCC are found in the recent past $(t>-20 \mathrm{Myr})$ for the other YLA. TW Hya was $62 \mathrm{pc}$ from LCC 8 Myr ago (the estimated age for this association), whereas the HD 141569 system was about 102 pc from LCC 5 Myr ago, continuously decreasing to 62 pc 18 Myr ago. The Tuc-Hor/GAYA association has maintained a distance to LCC of more than $100 \mathrm{pc}$ over the last $20 \mathrm{Myr}$, but this could be the only YLA considered here not to originate from a SN in LCC, since its estimated age is equal to (or even larger than) that derived for LCC.

We conclude that, at the moment of their birth, YLA were at distances of between 15 and $100 \mathrm{pc}$ from the centre of LCC, and even further from the other two Sco-Cen associations. Although observational errors in parallax and velocity components, as well as errors in age estimations, could affect these results, the fact that we work with mean values for distances, velocity components and ages minimises this possibility. Although the present radius of LCC is about $25 \mathrm{pc}$, it has been continuously expanding since birth, 16-20 Myr ago. Even considering a moderate expansion rate, one should expect an initial radius $\leqslant 20 \mathrm{pc}$. The distances obtained from Fig. 4, together with the expected reliability of the orbits, lead us to believe that the local associations were not born inside the cloud that formed the Sco-Cen complex, but in small molecular clouds outside it.

One possible scenario for the formation of YLA in these small molecular clouds is the explosion of one or several close SNe, which could have produced compression that triggered star formation. These hypothetical SNe should belong to the Sco-Cen complex. This complex is made up of several thousand stars, more than 300 of which are early-type stars, and around 35 are candidates for Type II SNe. Maíz-Apellániz (2001) estimated the number of past SNe inside the three associations, obtaining 1 SN for US, 13 for UCL and 6 for LCC. The first SN that exploded in each association took place when it was 
3-5 Myr old, and the others have been exploding and will continue to explode at a nearly constant rate, for the first $\sim 30$ Myr of the complex's life. Even a conservative estimate gives at least 6 SNe in UCL during the last 10-12 Myr, another 6 in LCC during the last 7-9 Myr and at least 1 in US.

The wave front of a $\mathrm{SN}$ typically moves at a velocity of a few tens of pc per million years; therefore, a SN explosion in LCC or UCL 9-11 Myr ago may have triggered star formation between 1 and 3 Myr later in small molecular clouds at distances of 15 to $75 \mathrm{pc}$. These would be the parent clouds of $\eta$ Cha and $\epsilon$ Cha, located at $\sim 20 \mathrm{pc}$ from LCC at their birth. Taking into account that the first SNe in LCC exploded when the associations were 3-5 Myr old (Maíz-Apellániz 2001), this scenario is only possible for a present age of LCC of at least $12 \mathrm{Myr}$. This is not a problem, since the estimated ages for LCC published in the literature range from 11-12 Myr to 16-20 Myr. If only one SN could explain this star formation outbreak $\sim 8.5-9$ Myr ago, this would be the age of $\eta$ Cha and $\epsilon$ Cha, which would have been formed simultaneously. It should be remembered that the estimated ages for $\eta$ Cha and $\epsilon$ Cha are $5-15$ and $\leqslant 10 \mathrm{Myr}$, respectively.

Such a SN could also have triggered the formation of TW Hya, whose estimated age is $\sim 8 \mathrm{Myr}$. As mentioned above, at that time TW Hya was about $45 \mathrm{pc}$ from the centre of LCC, in perfect agreement with the typical distance at which a SN wave front can trigger star formation in a small molecular cloud. However, it was not necessarily a single $\mathrm{SN}$ in LCC or UCL that was the origin of these four YLA. The SN rate in these two associations is $\sim 0.5 \mathrm{Myr}^{-1}$ and, therefore, it is possible that a few SNe in the period $-7 \leqslant t \leqslant-10$ Myr triggered the star formation that resulted in YLA. In any case, from our results we can conclude that these associations definitely did not form inside the associations of Sco-Cen, to be later ejected. They were formed in regions of space far from Sco-Cen, probably in small molecular clouds that were later totally dispersed by the newly born stars and/or by the shock fronts of later SNe in Sco-Cen.

Our results support a star formation scenario for very young stars far away from SFR or molecular clouds, such as that proposed by Feigelson (1996) and not that of Sterzik \& Durisen (1995). The latter authors perform numerical simulations to explain the existence of haloes of isolated T Tau stars around SFR. In their simulations a significant number of stars were ejected from these regions at birth with large velocities, allowing trajectories of some tens of pc in a few million years. Meanwhile, Feigelson (1996) proposed another scenario for the formation not only of the haloes of T Tau stars, but also of other completely isolated very young stars that have been discovered. In this model, the isolated T Tau stars form in small, fast-moving, short-lived molecular clouds. The gas remaining after the star formation process is rapidly dispersed by the stellar winds of the new stars. At present the stars are located in regions of space where there is no gas and so, apparently, they have formed far away from any SFR. The case of YLA supports this scenario, since our kinematic study shows that these associations formed far away from the Sco-Cen complex. For the HD 141569 system, a SN in UCL, as opposed to one in LCC, is a more promising candidate to explain its origin. This is because the distance to LCC for the range of ages accepted for this group (2-8 Myr) is between 88 and 116 pc, whereas for UCL it is $72-88 \mathrm{pc}$.

\subsection{Young local associations and Local Bubble}

If we superimposed on the present LB structure the trajectories back in time for YLA (see Fig. 13 in Fernández et al. 2008) we can see that the past orbits of the YLA are closer to the central region of the LB than Sco-Cen associations. To be exact, the trajectories of the centres of the associations TW Hya, Tuc-Hor/GAYA and $\beta$ Pic-Cap have crossed very near to the geometric centre of the LB in the last $~ 5$ Myr. The uncertainty boxes 


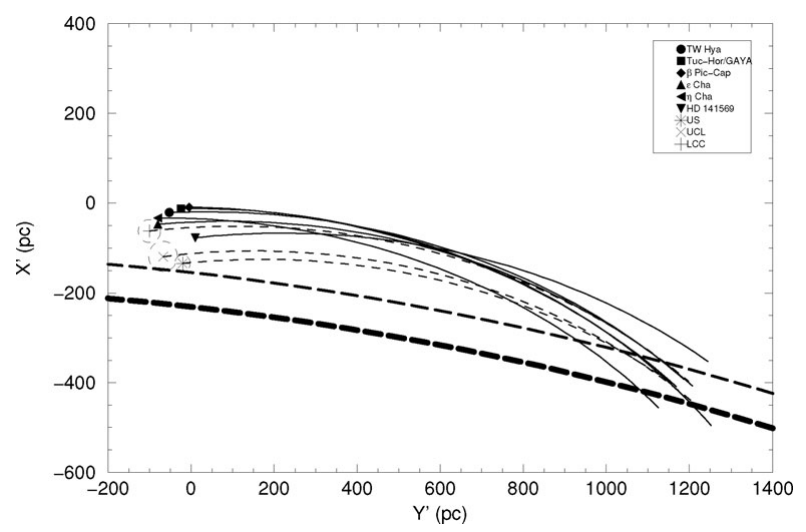

Figure 5. Orbits in the Galactic plane $\left(X^{\prime}-Y^{\prime}\right)$ integrated back in time to $t=-30 \mathrm{Myr}$ for YLA and the three associations of the Sco-Cen complex. The thick-dashed line shows the position of the minimum of the spiral potential (Fernández et al. 2001). The thin-dashed line is the position of the phase of the spiral structure $\psi=10^{\circ}$.

in position on the Galactic plane do not exceed a few tens of pc. On the other hand, errors in age estimates for the YLA result in positional errors along the trajectories back in time. Even considering the large uncertainties in age obtained for some of these YLA, ages are not expected to exceed $20 \mathrm{Myr}$ for any of them (except for Tuc-Hor/GAYA). We can therefore conclude that the YLA have been moving inside the LB for (at least) most of their lifetime, and can question whether the presence of these young stars inside the LB bears any relation to its origin and/or evolution.

All the YLA except TW Hya and $\beta$ Pic-Cap contain B-type stars (13 stars from a total of 223 members; see Table 6 in Fernández et al. 2008). It is not possible to derive the number of stars earlier than B2.5 that were born in the local associations, since we do not know their total mass precisely. However, the fact that at present we observe one SN candidate (a B2IV star), and more than a dozen stars of spectral types between B5 and B9, allows us to affirm that it is possible that one or more of these associations has sheltered a SN in the recent past (the last $10 \mathrm{Myr}$ ). As there is direct evidence for an explosion of a SN at a distance of $\sim 30 \mathrm{pc}, \sim 5 \mathrm{Myr}$ ago, several pieces of the same puzzle seem to support the theory of a recent SN in the nearest solar neighbourhood originating from a parent star belonging to a YLA, probably Tuc-Hor/GAYA or the extended R CrA association (which currently show the highest content of B-type stars). This near and recent $\mathrm{SN}$ would have been responsible for the reheating of the gas inside the LB needed to achieve the currently observed temperature of the diffuse soft X-ray background.

As we mention above, there is no agreement in the literature on the number of $\mathrm{SNe}$ needed to form the local cavity. If only one was enough, the SN we propose would be the most promising candidate, since it would be placed very near the geometric centre of the $\mathrm{LB}$, explaining in a natural way its present spatial structure. If more SNe are needed (as recent works suggest), we could consider other stars in the vicinity of LCC and UCL, as proposed by Fuchs et al. (2006).

\section{A scenario for the local and recent star formation}

If the impact of the spiral arm shock wave was the initial cause of star formation in the Sco-Cen region (see details in Fernández et al. 2008), then the history of the nearest solar neighbourhood during the last few tens of Myr would have been as follows. 30 Myr ago the GMC that became the parent of Sco-Cen was in the Galactic plane with coordinates 
$\left(X^{\prime}, Y^{\prime}\right) \sim(-400,1200)$ pc (see Fig. 5). The arrival of the potential minimum of the inner spiral arm triggered star formation in the region. At the same time it disturbed the cloud's motion, whose velocity vector became directed in the opposite direction to Galactic rotation and away from Galactic centre. The compression due to the spiral arm did not necessarily trigger star formation in the whole cloud, but perhaps only in the regions with the largest densities. This would be favoured by the smaller relative velocity between the shock wave and the RSR. The regions where star formation began must be those which generated UCL, LCC and, probably, the Tuc-Hor/GAYA association, which were all born at nearly the same time: about 16-20 Myr ago. The stellar winds from the first massive stars began to compress the gas of the neighbouring regions, maybe causing them to fragment into small molecular clouds that moved away from the central region of the parent cloud. About 9 Myr ago, a SN in LCC or UCL triggered star formation in these small molecular clouds, giving birth to the majority of YLA, as we saw in the previous section. The stellar winds of the newly born stars rapidly expelled the remaining gas from these small clouds (the cloudlets proposed by Feigelson 1996), completely erasing every trace of them and leading to our observation that there is no gas in these regions at present. YLA may have had a crucial influence on the history of the LB. We suggest that one or two SNe in these associations were responsible for reheating the LB a few million years ago. This hypothesis seems to be reinforced by the evidence of a very near SN about 5 Myr ago (Knie et al. 1999). At about the same time, as proposed by Preibisch \& Zinnecker (1999), the shock front of a SN in UCL would have triggered star formation in US about 6 Myr ago. Only 1.5 Myr ago, the most massive star in US would have gone $\mathrm{SN}$ and its shock front would now be reaching the molecular cloud of $\rho$ Oph, triggering the beginning of the star formation process there.

\section{References}

Asiain, R., Figueras, F., \& Torra, J. 1999, A\&A, 350, 434

Breitschwerdt, D. \& Cox, D. P. 2004, in: E. J. Alfaro, E. Pérez, \& J. Franco (eds.), How does the Galaxy work? A Galactic Tertulia with Don Cox and Ron Reynolds, Astrophysics and Space Science Library, vol. 315 (Dordrecht: Kluwer), p. 391

Feigelson, E. D. 1996, ApJ, 468, 306

Fernández, D., Figueras, F., \& Torra, J. 2001, A\&3A, 372, 833

Fernández, D., Figueras, F., \& Torra, J. 2008, A\&A, 480, 735

Fuchs, B., Breitschwerdt, D., de Avillez, M. A., Dettbarn, C., \& Flynn, C. 2006, MNRAS, 373, 993

Jilinski, E., Ortega, V. G., \& de la Reza, R. 2005, ApJ 619, 945

Knie, K., Korschinek, G., Faestermann, T., Wallner, C., Scholten, J., \& Hillebrandt, W. 1999, Phys. Rev. Lett., 81, 18

Lallement, R., Welsh, B. Y., Vergely, J. L., Crifo, F., \& Sfeir, D. M. 2003, A\& A, 411, 447

van Leeuwen, F. 2007, Hipparcos, the New Reduction of the Raw Data, Astrophysics and Space Science Library, vol. 350 (Dordrecht: Springer)

Madsen, S., Dravins, D., \& Lindegren, L. 2002, A\& $A$, 381, 446

Mamajek, E. E., Lawson, W. A., \& Feigelson, E. D. 2000, ApJ, 544, 356

Maíz-Apellániz, J. 2001, ApJ, 560, 83

Neuhäuser, R. \& Brandner, W. 1998, A\&A, 330, L29

Ortega, V. G., de la Reza, R., Jilinski, E., \& Bazzanella, B. 2004, ApJ, 609, 243

Preibisch, T. \& Zinnecker, H. 1999, AJ, 117, 2381

Sartori, M. J., Lépine, J. R. D., \& Dias, W. S. 2003, A\&\&A, 404, 913

Snowden, S. L., Egger, R., Finkbeiner, D. P., Freyberg, M. J., \& Plucinsky, P. P. 1998, ApJ, 493, 715

Sterzik, M. \& Durisen, R. 1995, A\&GA, 304, L9 
de Zeeuw, P. T., Hoogerwerf, R., Bruijne, J. H. J., Brown, A. G. A., \& Blaauw, A. 1999, AJ, 117,354

\section{Discussion}

MelBourne: What effect do these SNe have on the Earth? Are there tracers on Earth that suggest recent SNe explosions?

FERNÁNDEZ: In a paper published in 1999 by Knie and collaborators, they claimed to have found in a deep ocean crust evidences of the explosion of a $\mathrm{SN}$ in the recent past. They proposed that this SN exploded 5 Myr ago at a distance of about $30 \mathrm{pc}$.

MAMAJEK: Other researchers have calculated dynamical ages based on the time of minimum pass between some groups in the past. We are all here because we want improved ages for stars. How useful do you think these dynamical ages are?

FERNÁNDEZ: We have found that deriving dynamic ages is not a reliable aging methodology, al least considering the present membership assignment for each one of the associations. Other authors have derived dynamic ages, but using what they called "core stars"; that is, selecting those stars with the minimum dispersion in their velocity components. I strongly believe that the present membership assignment must be revised to be able to derive reliable dynamic ages for each one of these young local associations.

NAYLOR: You have a diagram which shows the motion of the local associations after they passed through the spiral arm. Where does the Sun move on this diagram? How long have we been near the local associations?

FERNÁnDEZ: We have not computed the orbit of the Sun in this figure, so I do not know exactly how long the Sun has been near the young local associations.

RoBerTO: Are the counts of low-mass stars in local associations compatible with the number of SNe?

FERnÁnDEZ: The problem with the counts of low-mass stars is that they are not complete and it is difficult to say if these counts are compatible with the number of SNe we propose (one or two). However, the counts of early-type stars, which are complete, seems to support the hypothesis that a SN exploded in the recent past in the young local associations. 


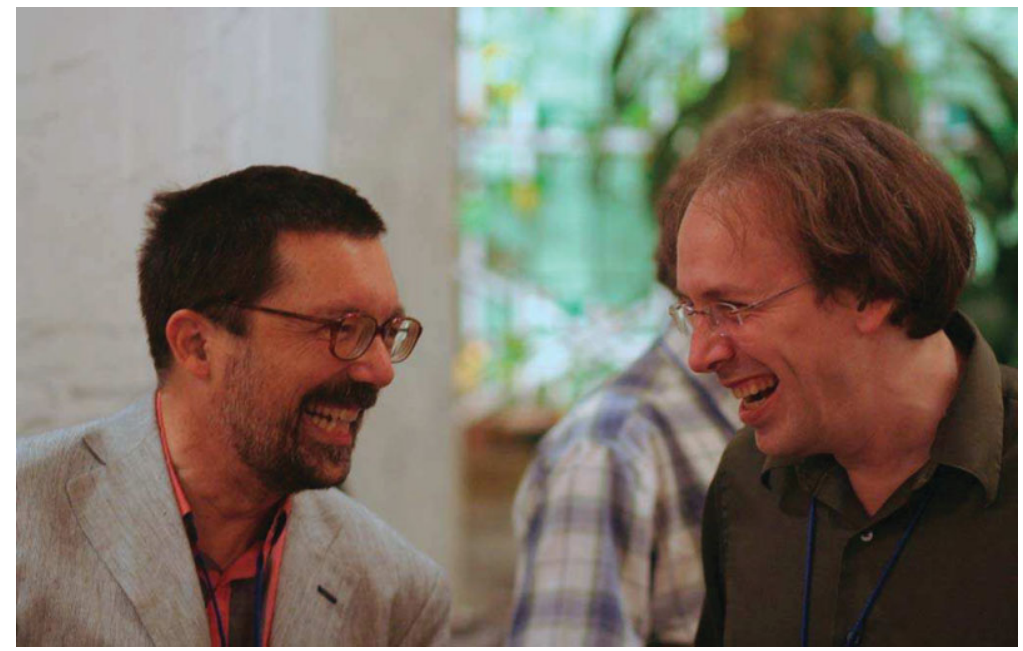

John Stauffer and Marc Pinsonneault

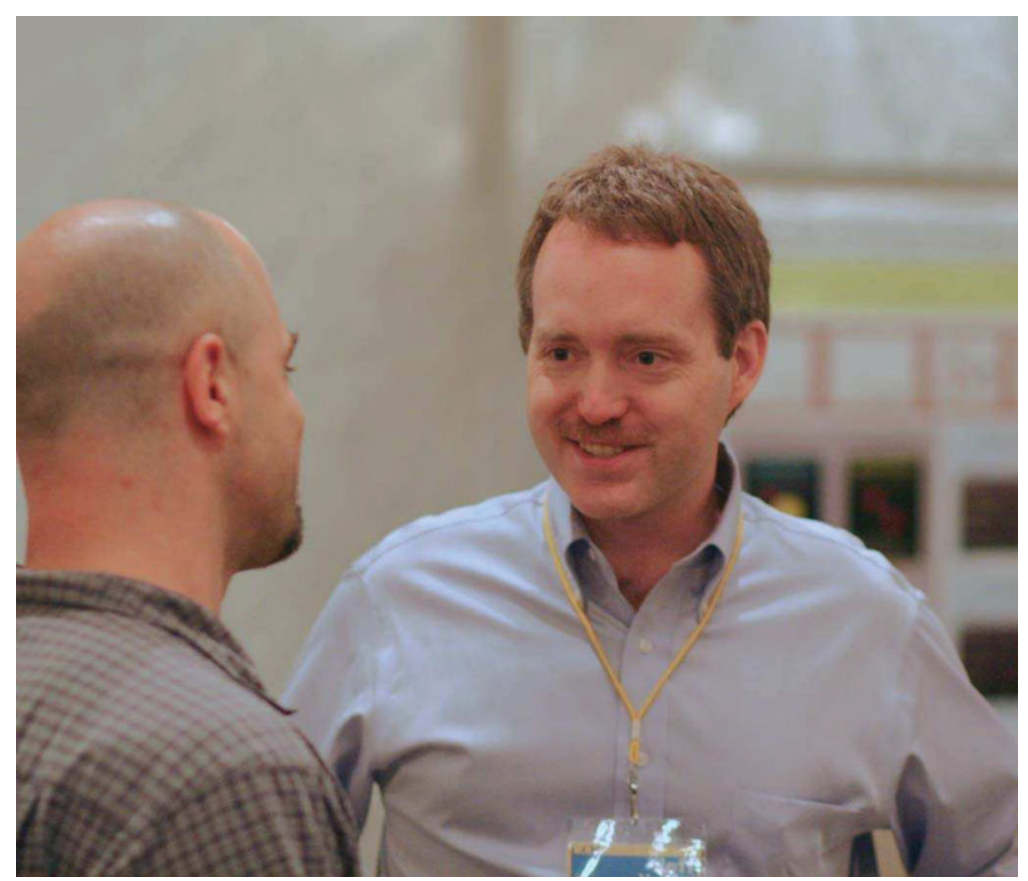

Jeff Valenti, LOC Chair 